\title{
Phenome-wide analysis highlights putative causal relationships between self-reported migraine and other complex traits
}

\author{
Luis M. García-Marín ${ }^{1,2}$ (B), Adrián I. Campos ${ }^{1,2}$, Nicholas G. Martin', Gabriel Cuéllar-Partida ${ }^{3,4}$ and \\ Miguel E. Rentería ${ }^{1,2^{*}}$
}

\begin{abstract}
Background: Migraine is a complex neurological disorder that is considered the most common disabling brain disorder affecting $14 \%$ of people worldwide. The present study sought to infer potential causal relationships between self-reported migraine and other complex traits, using genetic data and a hypothesis-free approach.

Methods: We leveraged available summary statistics from genome-wide association studies (GWAS) of 1,504 phenotypes and self-reported migraine and inferred pair-wise causal relationships using the latent causal variable (LCV) method.

Results: We identify 18 potential causal relationships between self-reported migraine and other complex traits. Hypertension and blood clot formations were causally associated with an increased migraine risk, possibly through vasoconstriction and platelet clumping. We observed that sources of abdominal pain and discomfort might influence a higher risk for migraine. Moreover, occupational and environmental factors such as working with paints, thinner or glues, and being exposed to diesel exhaust were causally associated with higher migraine risk. Psychiatric-related phenotypes, including stressful life events, increased migraine risk. In contrast, ever feeling unenthusiastic / disinterested for a whole week, a phenotype related to the psychological well-being of individuals, was a potential outcome of migraine.

Conclusions: Overall, our results suggest a potential vascular component to migraine, highlighting the role of vasoconstriction and platelet clumping. Stressful life events and occupational variables potentially influence a higher migraine risk. Additionally, a migraine could impact the psychological well-being of individuals. Our findings provide novel testable hypotheses for future studies that may inform the design of new interventions to prevent or reduce migraine risk and recurrence.
\end{abstract}

Keywords: Migraine, Genetics, Causal inference, Epidemiology, Complex traits

\section{Introduction}

Migraine is a complex neurological disorder characterised by intense, debilitating headaches on one side of

\footnotetext{
* Correspondence: miguel.renteria@qimrberghofer.edu.au

'Department of Genetics and Computational Biology, QIMR Berghofer Medical Research Institute, Brisbane, QLD, Australia

${ }^{2}$ School of Biomedical Sciences, Faculty of Medicine, The University of Queensland, Brisbane, QLD, Australia

Full list of author information is available at the end of the article
}

the head, and it is often accompanied by nausea, vomiting, numbness, and sensitivity to light and sound. Migraine is the most common disabling brain disorder, affecting $14 \%$ of the population [1-5].

A migraine episode is commonly composed of three stages $[1,3,6,7]$. First, during the prodromal phase, which can last from a couple of hours to several days, individuals may experience fatigue, lack of concentration,

C C The Author(s). 2021 Open Access This article is licensed under a Creative Commons Attribution 4.0 International License, which permits use, sharing, adaptation, distribution and reproduction in any medium or format, as long as you give appropriate credit to the original author(s) and the source, provide a link to the Creative Commons licence, and indicate if changes were made. The images or other third party material in this article are included in the article's Creative Commons licence, unless indicated otherwise in a credit line to the material. If material is not included in the article's Creative Commons licence and your intended use is not permitted by statutory regulation or exceeds the permitted use, you will need to obtain permission directly from the copyright holder. To view a copy of this licence, visit http://creativecommons.org/licenses/by/4.0/. The Creative Commons Public Domain Dedication waiver (http://creativecommons.org/publicdomain/zero/1.0/) applies to the data made available in this article, unless otherwise stated in a credit line to the data. 
excessive yawning, irritability, neck stiffness, light sensitivity, and nausea $[1,3,6,7]$. Then, in the headache phase, which can last a few hours or days, individuals are affected by strong headaches on one side of the head along with dizziness, light sensitivity, and vomiting $[1,3,6,7]$. Finally, the postdromal phase lasts a few hours or a day, and individuals are no longer afflicted by headache pain. Still, individuals can experience fatigue, irritability, nausea, light sensitivity, and lack of concentration [1, 3, 6, 7].

Migraine is a heritable trait, with heritability estimated between 30 and $60 \%$ [8]. Genome-wide association studies (GWAS) have led to substantial advances in understanding migraine's genetic aetiology. In particular, GWAS have identified over 40 genomic risk loci for migraine [9] and genetic overlap between migraine and other phenotypes, including attention-deficit hyperactive activity disorder (ADHD), major depressive disorder, epilepsy, stroke, and coronary artery disease [8]. Nonetheless, the identification of a shared genetic architecture between traits does not imply a causal relationship. A genetic correlation could be explained by vertical pleiotropic effects (i.e., a genetic variant's effect on a trait is mediated by its effect on another trait) or by horizontal pleiotropy (i.e., genetic variants have an effect on both traits). In particular, horizontal pleiotropy, and sample overlap, are known to bias estimates and increase the possibility of false-positive findings in genetic epidemiology studies using traditional methods, such as Mendelian randomisation, to assess causality $[10,11]$.

As an alternative to Mendelian randomisation, the latent causal variable method (LCV) was developed to provide an opportunity to investigate potential causal associations with a different approach (see Methods) [10]. Advantages of the LCV method include that it is able to minimise the effects of sample overlap [10, 12-14], it is less susceptible to confounding by horizontal pleiotropy $[10,12]$, and it uses aggregated genetic information throughout the entire genome to increase statistical power, allowing to test "underpowered" phenotypes with none or few significant genome-wide loci [10, 12-14].

Despite extensive efforts to understand migraine's aetiology, there is still a growing need to generate investigation lines that can successfully incorporate results and hypotheses from genetic studies of migraine into clinical practice. That could enable the identification of diseasemodifying traits and pinpoint the underlying causes of this complex neurological disorder. Here, we conduct a multitrait analysis of GWAS with samples for self-reported migraine in the 23andMe cohort and chronic headaches in the UK Biobank. Then, we leverage an extensive collection $(N=1,504)$ of GWAS summary statistics to conduct a hypothesis-free phenome-wide screening of traits causally associated with migraine using the latent causal variable (LCV) method. Our results generate testable hypotheses for future studies and provide novel insights into the relationship between migraine and overall health.

\section{Methods \\ Sample}

The present study used samples from the 23andMe and UK Biobank cohorts. Details are given below.

\section{3andMe cohort}

The 23andMe cohort comprised 30,465 self-reported migraine cases and 143,147 controls and was previously included in the meta-analysis by Gormely et al. [15]. Participants provided informed consent and reported their migraine diagnosis. Covariates included sex, age, and five principal genetic components.

\section{UK Biobank cohort}

We used information for chronic headaches as a proxy trait for migraine, as several cases of migraine might have been undiagnosed or lacking an ICD10 code. Chronic headaches were defined as pain in the head lasting for at least three months. In total, 39,283 ( 9\%) of the UK Biobank participants included in the analysis were considered cases for chronic headaches.

Chronic headache was defined using the item "Have you had headaches for more than three months?" (FieldID: 3799), which could be answered with "Yes", "No", "Do not know", or "Prefer not to answer". Individuals that selected "Yes" were defined as cases, whereas those who selected "No" for headaches for more than three months were defined as controls. GWAS was performed using REGENIE (v1.0.6.2), a method that implements a logistic mixed-effects model. This approach models genetic relationships between individuals as a random effect to account for cryptic relatedness. Quality control consisted of excluding variants with minor allele frequency $(\mathrm{MAF})<0.005$, imputation quality $<0.6$ and those deviating from Hardy-Weinberg equilibrium ( $p$-value $<1 \times$ $10^{-5}$ ). After quality control exclusions, a total of 11,172 , 285 single nucleotide polymorphisms (SNPs) remained in the analysis. Subject data were excluded if their genotype-derived principal components 1 and 2 were further than six standard deviations away from $1000 \mathrm{Ge}-$ nomes European sample population. Sex, age, genotyping array, and the top 10 principal components derived from genetic data were used as covariates for the association analysis. After quality control, a total of up to 441, 088 individuals remained in the GWAS.

\section{GWAS Multivariate analysis}

Given that chronic headaches and self-reported migraine represent highly related but not the same trait, we performed a multi-trait analysis of GWAS (MTAG) analysis [16]. This approach leverages LD-score regression [17] 
to estimate a genetic correlation between two GWAS and perform a weighted meta-analysis and boost statistical power. We used MTAG(v2019/08/26) to combine the 23andMe self-reported migraine GWAS summary statistics with the UK-Biobank chronic headaches GWAS and observed a robust genetic correlation $\left(\mathrm{r}_{\mathrm{G}}=\right.$ 0.83 s.e. $=0.05$ ). Because the current study's scope is migraine, we focused on the output specific to migraine, which had $>35$ genome-wide significant loci.

\section{Datasets}

The Complex Traits Genomics Virtual Lab (CTG-VL; https://genoma.io/) [18] has made available a compilation of GWAS summary statistics for 1,504 traits. A substantial proportion of these correspond to the second wave of GWAS results from the UK Biobank released by the Neale Lab (www.nealelab.is/uk-biobank/) [19], and the rest come from multiple GWAS consortia. Thus, most GWAS were performed using European ancestry individuals, and traits include objective laboratory measurements, self-reported phenotypes, and consortia meta-analyses. UK Biobank GWAS were adjusted for age, age-squared, inferred sex, age * inferred sex, agesquared * inferred sex, and 20 genetic ancestry principal components $[18,19]$. For the present study, only GWAS derived from European populations were used to avoid biases due to population differences in linkagedisequilibrium and allele frequencies [18].

\section{Genetic correlations}

A genetic correlation indicates the extent to which genetic effect sizes at common genetic variants are shared between two different phenotypes [13, 20]. We performed genetic correlation analyses using an offline version of CTG-VL for the LCV method, in which a linkage disequilibrium score regression [17] is used to assess a genetic correlation between two traits. Multiple testing was corrected for using Benjamini-Hochberg's False Discovery Rate (FDR $<5 \%)$.

\section{Genetic causal proportion}

We estimated the genetic causal proportion (GCP) between migraine and 1,504 other phenotypes using the phenome-wide analysis pipeline in the CTG-VL as described in previous studies [12-14] to assess if a significant genetic correlation could be explained by a potential causal relationship between the two traits. Shortly, the CTG-VL implemented the phenome-wide LCV analysis pipeline in $\mathrm{R} 4.0 .0$ [12] based on the $\mathrm{R}$ script that the original authors of the LCV method [10] have made available in the Github repository (https:// github.com/lukejoconnor/LCV). Further, to ensure consistency of alleles and variants across GWAS summary statistics, data was formatted using munge sumstats.py, which is available by the LD-score software and extracted hapmap SNPs using the provided list of SNPs (w_hm3.snplist) (https://github.com/bulik/ldsc/ wiki). In the present study, we uploaded GWAS summary statistics for migraine onto CTG-VL. Then, we performed the phenome-wide analysis pipeline to estimate genetic correlations and GCP estimates with LDscore regression and LCV, respectively. Finally, we used causal architecture plots to illustrate our results. A detailed and illustrated description of this approach is available in previous studies $[12,13]$.

Regarding correction for multiple comparisons, we applied the LCV method to estimate the GCP for all phenotypes with evidence of a genetic correlation with selfreported migraine at Benjamini-Hochberg's False Discovery Rate $(\mathrm{FDR}<5 \%)$. Then, $\mathrm{FDR}<5 \%$ was used to account for multiple comparisons and identify those traits that had evidence of a potential causal relationship based on their GCP.

LCV leverages GWAS summary statistics to estimate a genetic correlation and relies on a latent variable $L$, assumed to be the causal constituent mediating the genetic correlation between both traits, to estimate the genetic causality proportion (GCP) $[10,12,13,21]$. An absolute GCP value of 1 indicates the detection of a causal association that could be explained by vertical pleiotropic effects among a pair of genetically correlated phenotypes (i.e., the effect of a genetic variant on a trait is mediated by its effect on another trait). In contrast, a GCP value of zero implies horizontal pleiotropic effects between the phenotypes, in which case an intervention on any of them would not affect the other, a consequence of the absence of genetic causality between them $[10,12,13]$. Also, a $|\mathrm{GCP}|<0.60$ is considered low and indicates limited partial genetic causality [10]. Multiple testing was corrected for using Benjamini-Hochberg's False Discovery Rate (FDR $<5 \%)$.

\section{Results}

We identified 510 significant genetic correlations of which 17 were found to be potential causal associations with self-reported migraine $(|\mathrm{GCP}|>0.60 ; \mathrm{FDR}<5 \%$; Supplementary File 1) and one showed evidence of limited partial genetic causality $(|\mathrm{GCP}|<0.60$; FDR $<5 \%$; Supplementary File 1).

Traits identified to potentially increase self-reported migraine risk include phenotypes allocated in the International Classification of Diseases (ICD10) such as diaphragmatic hernia $\left(\mathrm{r}_{\mathrm{G}}=0.53, \mathrm{GCP}=-0.77, \mathrm{GCP}_{\text {pvalue }}=\right.$ $\left.4.85 \times 10^{-06}\right)$, ventral hernia $\left(\mathrm{r}_{\mathrm{G}}=0.27, \mathrm{GCP}=-0.68\right.$, $\left.\mathrm{GCP}_{\text {pvalue }}=7.04 \times 10^{-04}\right)$, and benign neoplasm of colon, rectum, anus and anal canal $\left(\mathrm{r}_{\mathrm{G}}=0.19, \mathrm{GCP}=-0.71\right.$, $\left.\mathrm{GCP}_{\text {pvalue }}=2.91 \times 10^{-08}\right)($ Fig. 1 ; Table 1$)$. 

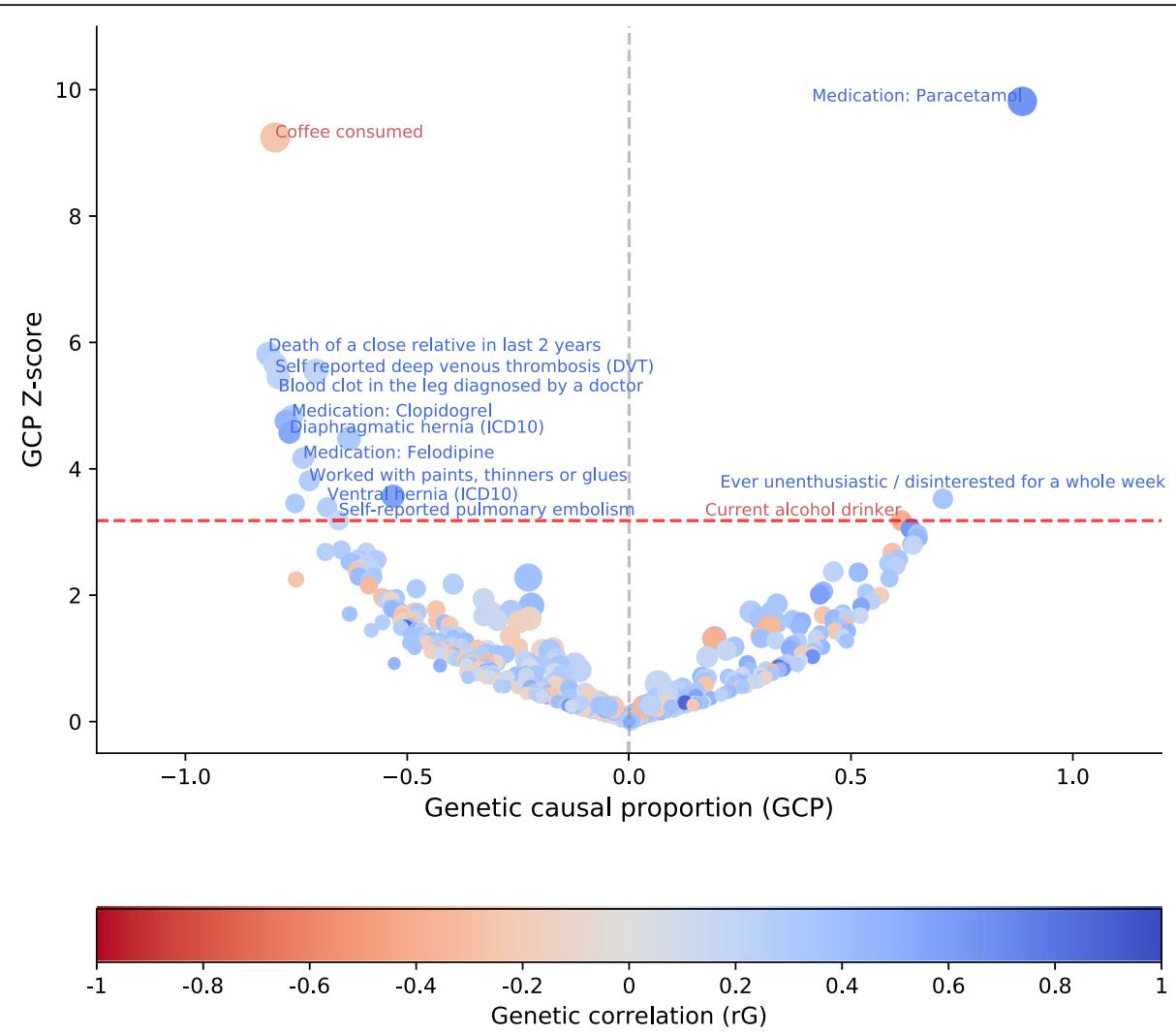

Fig. 1 Potentially causal associations for migraine. Causal architecture plots illustrating the latent causal variable exposome-wide analysis results. Each dot represents a trait with a significant genetic correlation with migraine. The $y$-axis shows the genetic causality proportion (GCP) absolute Z-score (statistical significance), whilst the $x$-axis exhibits the GCP estimate. The red dashed lines represent the statistical significance threshold $(\mathrm{FDR}<5 \%$ ), while the division for traits causally influencing migraine (on the left) and traits causally influenced by migraine (on the right) is represented by the grey dashed lines. Phenotypes in blue show a positive genetic correlation with migraine, while phenotypes in red show a negative genetic correlation with migraine

Our results show that self-reported migraine influences an increase in the intake of the analgesic Paracetamol $\left(\mathrm{r}_{\mathrm{G}}=0.66, \mathrm{GCP}=0.77, \mathrm{GCP}_{\text {pvalue }}=9.70 \times 10^{-23}\right)$. In contrast, a higher self-reported migraine risk is influenced by traits involving the use of various medications such as, Clopidogrel, which is an antiplatelet drug, and Felodipine commonly prescribed for hypertension (Fig. 1; Table 1).

Self-reported cardiovascular phenotypes such as deep venous thrombosis $(D V T)\left(\mathrm{r}_{\mathrm{G}}=0.21, \mathrm{GCP}=-0.80\right.$, $\left.\mathrm{GCP}_{\text {pvalue }}=1.59 \times 10^{-08}\right)$ and pulmonary embolism $\left(\mathrm{r}_{\mathrm{G}}\right.$ $\left.=0.19, \mathrm{GCP}=-0.65, \mathrm{GCP}_{\text {pvalue }}=1.45 \times 10^{-03}\right)$ appear to increase self-reported migraine risk, as do some phenotypes diagnosed by a doctor, including a blood clot in $\operatorname{leg}\left(\mathrm{r}_{\mathrm{G}}=0.19, \mathrm{GCP}=-0.79, \mathrm{GCP}_{\text {pvalue }}=5.15 \times 10^{-08}\right)$ and stroke $\left(\mathrm{r}_{\mathrm{G}}=0.32, \mathrm{GCP}=-0.63, \mathrm{GCP}_{\text {pvalue }}=7.55 \times\right.$ $10^{-06}$ ) (Fig. 1; Table 1).

For psychiatric-related phenotypes, self-reported migraine was a potential outcome of stressful life events such as death of a close relative in the last two years $\left(_{\mathrm{G}}\right.$ $=0.27, \mathrm{GCP}=-0.81, \mathrm{GCP}_{\text {pvalue }}=6.00 \times 10^{-09}$ ), whilst ever feeling unenthusiastic / disinterested for a whole week $\left(\mathrm{r}_{\mathrm{G}}=0.53, \mathrm{GCP}=0.70, \mathrm{GCP}_{\text {pvalue }}=4.25 \times 10^{-04}\right)$ was a putative migraine consequence.

Occupational-related variables such as worked with paint, thinner or glues $\left(\mathrm{r}_{\mathrm{G}}=0.28, \mathrm{GCP}=-0.72, \mathrm{GCP}_{\text {pvalue }}\right.$ $=1.39 \times 10^{-04}$ ) and being at a workplace that had a lot of diesel exhaust $\left(\mathrm{r}_{\mathrm{G}}=0.25, \mathrm{GCP}=-0.81, \mathrm{GCP}_{\text {pvalue }}=\right.$ $3.69 \times 10^{-04}$ ) potentially increase self-reported migraine risk (Fig. 1; Table 1).

\section{Discussion}

The present study contributes to advance the understanding of migraine's aetiology by providing insights into migraine's causal architecture. We used GWAS data to investigate potential causal associations between selfreported migraine and 1,504 different phenotypes and identified 18 inferred causal associations. Overall, our results show a putative vascular component to selfreported migraine and suggest that abdominal discomfort and stressful life events could increase self-reported migraine risk.

Abdominal discomfort has been previously associated with migraine [22-24]. For example, it has been shown 
Table 1 Traits with an inferred causal relationship with migraine

\begin{tabular}{|c|c|c|c|c|c|c|}
\hline Trait & GCP & GCP se & GCP pval & $r_{G}$ & $r_{G}$ se & $\mathrm{r}_{\mathrm{G}}$ pval \\
\hline Medication: Paracetamol & 0.89 & 0.09 & $9.70 \mathrm{E}-23$ & 0.66 & 0.03 & $2.75 \mathrm{E}-90$ \\
\hline Coffee consumed & -0.80 & 0.09 & $2.40 \mathrm{E}-20$ & -0.25 & 0.10 & $1.36 \mathrm{E}-02$ \\
\hline Death of a close relative in last 2 years & -0.81 & 0.14 & $6.00 \mathrm{E}-09$ & 0.27 & 0.06 & $3.55 \mathrm{E}-05$ \\
\hline Self reported deep venous thrombosis (DVT) & -0.80 & 0.14 & 1.59E-08 & 0.21 & 0.07 & 2.61E-03 \\
\hline Benign neoplasm of colon, rectum, anus and anal canal (ICD10) & -0.71 & 0.13 & 2.91E-08 & 0.19 & 0.05 & $5.30 \mathrm{E}-04$ \\
\hline Blood clot in the leg diagnosed by a doctor & -0.79 & 0.15 & $5.15 \mathrm{E}-08$ & 0.19 & 0.07 & $6.22 \mathrm{E}-03$ \\
\hline Medication: Clopidogrel & -0.76 & 0.16 & $1.38 \mathrm{E}-06$ & 0.27 & 0.11 & $1.48 \mathrm{E}-02$ \\
\hline Medication: Dihydrocodeine & -0.77 & 0.16 & $1.98 \mathrm{E}-06$ & 0.47 & 0.16 & $3.75 \mathrm{E}-03$ \\
\hline Diaphragmatic hernia (ICD10) & -0.77 & 0.17 & 4.85E-06 & 0.53 & 0.08 & $6.45 \mathrm{E}-11$ \\
\hline Stroke diagnosed by doctor & -0.63 & 0.14 & 7.55E-06 & 0.32 & 0.11 & 3.83E-03 \\
\hline Medication: Felodipine & -0.73 & 0.18 & $3.06 \mathrm{E}-05$ & 0.27 & 0.09 & 2.03E-03 \\
\hline Worked with paints, thinners or glues & -0.72 & 0.19 & 1.39E-04 & 0.28 & 0.09 & $1.71 \mathrm{E}-03$ \\
\hline Ever unenthusiastic / disinterested for a whole week & 0.71 & 0.20 & 4.25E-04 & 0.34 & 0.05 & $8.12 \mathrm{E}-11$ \\
\hline Workplace sometimes had a lot of diesel exhaust & -0.75 & 0.22 & $5.55 \mathrm{E}-04$ & 0.31 & 0.08 & 2.44E-04 \\
\hline Ventral hernia (ICD10) & -0.68 & 0.20 & 7.04E-04 & 0.27 & 0.10 & $8.35 \mathrm{E}-03$ \\
\hline Self-reported pulmonary embolism & -0.65 & 0.21 & $1.45 \mathrm{E}-03$ & 0.19 & 0.07 & 1.13E-02 \\
\hline Current alcohol drinker & 0.61 & 0.19 & 1.47E-03 & -0.39 & 0.04 & $1.51 \mathrm{E}-19$ \\
\hline
\end{tabular}

This table shows all traits with a significant $(\mathrm{FDR}<5 \%)$ and strong genetic causal proportion $(|\mathrm{GCP}|>0.60)$ with migraine. Due to space constraints, results for all nominally significant genetic correlations for migraine are shown in Supplementary File 1.

Trait Trait causally associated with obesity, GCP Genetic causal proportion, GCP se Genetic causal proportion standard deviation, GCP pval Genetic causal proportion unadjusted $p$-value, $r_{G}$ Genetic correlation, $r_{G}$ se Genetic correlation standard deviation, $r_{G} p$ val Genetic correlation unadjusted $p$-value

that migraine among patients with abdominal discomfort can be eased once the abdominal pain source is treated $[22,23]$. Thus, a plethora of studies seeks to determine whether gastrointestinal factors could influence the development of a migraine episode. However, the range of gastrointestinal abnormalities and disorders related to migraine has not been entirely disclosed. In the present study, phenotypes such as diaphragmatic hernia (ICD10), ventral hernia (ICD10), and benign neoplasm of colon, rectum, anus and anal canal (ICD10), which are known to be usually accompanied by inflammation and abdominal pain or discomfort [25-28], posed a putative causal effect increasing self-reported migraine risk. Therefore, our results are aligned with the hypothesis in which abdominal pain could be a risk factor for migraine, possibly through inflammatory or vascular mediators [23]. These findings could be used as testable hypotheses in future studies, which in turn should seek to describe the possible molecular underpinnings underlying the relationship between migraine and sources of abdominal pain or discomfort.

Previous research has aimed to describe the extent to which vascular components explain migraine [29-31]. Mendelian randomisation studies have identified diastolic blood pressure as potentially causal for migraine [32]. In our study, self-reports of pulmonary embolism and deep venous thrombosis and traits diagnosed by a doctor such as a blood clot in the leg increased selfreported migraine risk. Similarly, the use of medications for cardiovascular disorders was also found to increase the risk for self-reported migraine. For instance, Felodipine could be used as a proxy for hypertension [33], while Clopidogrel could be used as a proxy for blood clots and thromboembolisms [34]. Felodipine is a calcium-channel blocker used to treat hypertension by blocking calcium ions' entry into the cell and minimising vascular smooth muscle contraction [33]. This mechanism has incremented calcium-channel blockers use as an off-label migraine medication [35]. In addition, clopidogrel is an antiplatelet drug commonly prescribed for thromboembolism prevention [36], and previous studies have demonstrated platelet aggregations to be significantly higher in individuals during a migraine episode $[37,38]$. Therefore, our results suggest a potential vascular component to migraine, supporting the hypothesis in which vascular changes lead to migraine. We speculate that this relationship could be mediated by increased platelet clumping and vasoconstriction, perhaps as a consequence of hypertension and blood clots formation.

Lower socioeconomic status (SES) has been associated with a higher risk for chronic diseases [39, 40]. For instance, it has been noted that individuals with low SES are more likely to be exposed to hazardous substances at their workplace [39, 40]. In our study, self-reported 
migraine was a putative outcome of work environments in which individuals work with paint, thinner or glues, or are in a workplace with a lot of diesel exhaust. In contrast, other work environments, such as being in a workplace often full of chemicals or other fumes, did not show evidence for a potential causal association with selfreported migraine (Supplementary File 1). These results are consistent with previous studies suggesting that headaches and chronic neurological symptoms are prevalent among individuals exposed to mixture solvents and can be explained by the acute effect of solvents in the central nervous system [41, 42]. Similarly, it has also been reported that exposure to diesel exhaust particles is associated with neuroinflammation and headaches [4345]. Although we cannot rule out the potential influence of SES-related variables such as educational attainment and assortative mating in the relationship between work environments and migraine. Our results are consistent with previous observational studies, suggesting that migraine could be an outcome of being exposed to diesel exhaust and working with solvents due to neuroinflammation and hazardous effects in the central nervous system.

The relationship between migraine and emotional distress has been described before; however, it has not been fully elucidated. For instance, several studies point out that migraine is associated with psychiatric disorders such as depression and anxiety [46, 47], while others suggest that the duration of a migraine episode is related to an impairment of anger control [48]. In the present study, experiencing the death of a close relative in the last two years, which is considered a major stressful life event that can lead to depression or anxiety [49], was potentially influencing an increase in self-reported migraine. In contrast, ever feeling unenthusiastic / disinterested for a whole week was identified as a putative consequence of self-reported migraine. Thus, our results show that stressful life events can be a risk factor for migraine, while migraine holds an impact on the psychological well-being of individuals, which in turn suggests that identifying psychological vulnerabilities among migraineurs is of great importance.

We note that statistical methods used in genetic epidemiology such as LCV and Mendelian randomisation test the potential causal effect of the genetic liability for a disease on the outcome [32]. Migraine can have its onset at around the age of 20 , and some phenotypes identified to be causally associated with self-reported migraine can have a late-onset (i.e., stroke diagnosed by a doctor and neoplasms). Since the present study could not take into account the age of onset of migraine and other conditions, it is likely that genetic variants associated with late-onset phenotypes increase the risk for self-reported migraine, rather than the phenotype itself. Therefore, causal associations between self-reported migraine and late-onset phenotypes must be interpreted with caution.

We highlight the importance of triangulating results from multiple study designs, of which at least one should be an interventional study (i.e., a randomised controlled trial). However, interventional studies can be expensive, time-consuming, or unethical to perform. Therefore, identifying potential causal associations using statistical methods in genetic epidemiology could be the best option available. We suggest that the inferred causal relationships identified through LCV analyses should be testable hypotheses for future observational and genetic studies.

Important limitations of this study must be acknowledged. For instance, given that previous studies have highlighted racial and ethnic differences in migraine [50], and our analyses used participants of European ancestry, the generalisability of our results outside European ancestry individuals may be limited. Moreover, although more than 1500 traits were included in our analyses, causal associations with other traits may exist. Also, even though the LCV method uses aggregated information across the genome to increase statistical power, the GCP estimate still relies on the statistical power of GWAS [10, 12, 13], limiting the capability to infer causal effects for some traits. In addition, the presence of multiple latent factors could reduce the statistical power of the analysis and lower GCP estimates [10]. Lastly, since the bivariate nature of the LCV method aims to identify the predominant causal pathway between a pair of genetically correlated phenotypes [10], bidirectional causality between traits cannot be tested.

It is fundamental to consider potential biases in the design of the GWAS involved. Although the selfreported migraine sample used in the present study has been previously included in a large migraine metaanalysis, comprising $\sim 52 \%$ of the cases which allowed the identification of $\sim 40$ independent loci associated with migraine [15], we note that using self-reported data may have led to a recruitment bias since some patients may have misdiagnosed themselves with migraine [51]. Nonetheless, it has been reported that self-reported migraine can be congruent with a clinical diagnosis. Still, a self-report of migraine without a clinical diagnosis criteria is not able to delineate between subtypes such as migraine with or without an aura [52]. Similarly, we broadly define chronic headaches with the standard question of the International Headache Society [53] "Have you had headaches for more than three months?", which could apply to primary or secondary types of headaches. As we mention in the methods section, since the focus of this study is migraine, we only used chronic headaches to boost statistical power for self-reported migraine due to the strong genetic correlation between both phenotypes $\left(\mathrm{r}_{\mathrm{G}}=0.83\right.$ s.e. $\left.=0.05\right)$. 
Future studies should aim to assess whether the potential causal associations identified in the present study could be specific to a particular type of migraine and if they can be observed for secondary type headaches. As previous studies have noted [54], we suggest that medication use GWAS in this study should be interpreted as a proxy for the disease or disorder requiring the medication; however, as we mentioned earlier, due to the nature of self-reported data, the identification of migraine comorbidities only through medication use GWAS could be inaccurate and should be addressed with caution until confirmed by future studies. Related to this is the interpretation of medication use Felodipine, a proxy for hypertension, which could be indicative of a putative causal association between hypertension and secondarytype headache.

\section{Conclusions}

We provide evidence for potential causal relationships between self-reported migraine and 1,504 different phenotypes. Our findings uncovered changes in blood vessels, particularly vasoconstriction and platelet clumping, potentially increasing migraine risk. Further, we show the putative influence of workplace environments increasing migraine risk and reveal emotional stress as a risk factor for migraine. Also, we reveal migraine's potential impact on the psychological well-being of individuals. In addition, we raise the possibility of abdominal pain influencing a higher migraine risk. Altogether, our results confirm some causal associations contemplated in previous studies and point out testable hypotheses that, if confirmed, may open novel avenues in the understanding of migraine, which in turn can provide opportunities to improve the design of emerging treatments and drug targets.

\section{Supplementary Information}

The online version contains supplementary material available at https://doi. org/10.1186/s10194-021-01284-w.

Additional file 1: Supplementary File 1. LCV output for migraine.

\footnotetext{
Acknowledgements

We thank the participants and the research team of 23andMe for making the present work possible.

This article has been selected as the winner of the 2021 Greppi Award. The Enrico Greppi Award is made to an unpublished paper dealing with clinical, epidemiological, genetic, pathophysiological or therapeutic aspects of headache. The Italian Society for the Study of Headached (SISC) sponsors this award. This article has undergone the journal's standard peer-review process overseen by the Editor-in-Chief. The members of the 2021 Enrico Greppi Award Selection Committee were: Paolo Calabresi, Gianluca Coppola, Zaza Katsarava, Paolo Martelletti, Cristina Tassorelli.
}

\section{Authors' contributions}

M.E.R. and L.M.G.M. conceived and directed the study. L.M.G.-M. performed the statistical and bioinformatics analyses, with support and input from A.I.C., N.G.M., G.C.P., and M.E.R. L.M.G.-M. wrote the first draft of the paper and integrated input and feedback from all co-authors. The author(s) read and approved the final manuscript.

\section{Funding}

AIC and LMGM are supported by UQ Research Training Scholarships from The University of Queensland (UQ). MER thanks the support of the National Health and Medical Research Council and Australian Research Council through a Research Fellowship (APP1102821).

\section{Availability of data and materials}

Individual-level data for UK Biobank participants are available to eligible researchers through the UK Biobank (www.biobank.ac.uk). Individual-level data for 23andMe are available upon request through a Data Transfer Agreement and the appropriate application procedure (https://research.23andme.com/ dataset-access/).

\section{Declarations}

Ethics approval and consent to participate

All participants provided informed consent. This study was approved by the Human Research Ethics Committee of the QIMR Berghofer Medical Research Institute.

Consent for publication

Not applicable.

\section{Competing interests}

GC-P contributed to this study while employed at The University of Queensland. He is now an employee of 23andMe Inc. and he may hold stock or stock options. All other authors declare having no conflicts of interest.

\section{Author details}

'Department of Genetics and Computational Biology, QIMR Berghofer Medical Research Institute, Brisbane, QLD, Australia. ${ }^{2}$ School of Biomedical Sciences, Faculty of Medicine, The University of Queensland, Brisbane, QLD, Australia. ${ }^{3}$ The University of Queensland Diamantina Institute, The University of Queensland, Woolloongabba, QLD, Australia. ${ }^{4}$ Present address: 23andMe, Inc, Sunnyvale, California, USA.

Received: 3 May 2021 Accepted: 18 June 2021

Published online: 08 July 2021

\section{References}

1. Genetics Home Reference Migraine. https://ghr.nlm.nih.gov/condition/migra ine. Accessed 31 Jul 2020

2. Victor TW, Hu X, Campbell JC et al (2010) Migraine prevalence by age and sex in the United States: a life-span study. Cephalalgia 30:1065-1072. https://doi.org/10.1177/0333102409355601

3. Kikkeri NS, Nagalli S (2020) Migraine with Aura. In: StatPearls. Treasure Island, StatPearls Publishing

4. GBD 2016 Headache Collaborators (2018) Global, regional, and national burden of migraine and tension-type headache, 1990-2016: a systematic analysis for the Global Burden of Disease Study 2016. Lancet Neurol 17:954976. https://doi.org/10.1016/S1474-4422(18)30322-3

5. Weatherall MW (2015) The diagnosis and treatment of chronic migraine. Ther Adv Chronic Dis 6:115-123. https://doi.org/10.1177/2040622315579627

6. Bahra A (2011) Primary Headache Disorders: Focus on Migraine. Rev Pain 5: 2-11. https://doi.org/10.1177/204946371100500402

7. Boran HE, Bolay H (2013) Pathophysiology of Migraine. Noro Psikiyatr Ars 50: S1-S7. https://doi.org/10.4274/Npa.y7251

8. Sutherland $H G$, Albury CL, Griffiths LR (2019) Advances in genetics of migraine. J Headache Pain 20:1-20. https://doi.org/10.1186/s10194-019-101 7-9

9. van den Maagdenberg AMJM, Nyholt DR, Anttila V (2019) Novel hypotheses emerging from GWAS in migraine? J Headache Pain 20:5. https://doi.org/1 0.1186/s10194-018-0956-x

10. O'Connor LJ, Price AL (2018) Distinguishing genetic correlation from causation across 52 diseases and complex traits. Nat Genet 50:1728-1734. https://doi.org/10.1038/s41588-018-0255-0 
11. Koellinger PD, de Vlaming $R$ (2019) Mendelian randomization: the challenge of unobserved environmental confounds. Int J Epidemiol 48:665-671. https://doi.org/10.1093/ije/dyz138

12. Haworth S, Kho PF, Holgerson PL et al (2020) Assessment and visualization of phenome-wide causal relationships using genetic data: an application to dental caries and periodontitis. Eur J Hum Genet. https://doi.org/10.1038/ s41431-020-00734-4

13. García-Marín LM, Campos Al, Martin NG et al (2020) Inference of causal relationships between sleep-related traits and 1,527 phenotypes using genetic data. Sleep. https://doi.org/10.1093/sleep/zsaa154

14. García-Marín LM, Campos Al, Kho P-F et al (2021) Phenome-wide screening of GWAS data reveals the complex causal architecture of obesity. Hum Genet. https://doi.org/10.1007/s00439-021-02298-9

15. Gormley P, Anttila V, Winsvold BS et al (2016) Meta-analysis of 375,000 individuals identifies 38 susceptibility loci for migraine. Nat Genet 48:856866. https://doi.org/10.1038/ng.3598

16. Turley P, Walters RK, Maghzian O et al (2018) Multi-trait analysis of genomewide association summary statistics using MTAG. Nat Genet 50:229-237. https://doi.org/10.1038/s41588-017-0009-4

17. Bulik-Sullivan BK, Loh P-R, Finucane HK et al (2015) LD Score regression distinguishes confounding from polygenicity in genome-wide association studies. Nat Genet 47:291-295. https://doi.org/10.1038/ng.3211

18. Cuéllar-Partida G, Lundberg M, Kho PF et al (2019) Complex-Traits Genetics Virtual Lab: A community-driven web platform for post-GWAS analyses. bioRxiv 518027. https://doi.org/10.1101/518027

19. Neale's Lab (2018) GWAS Results. In: UK Biobank—Neale Lab. http://www. nealelab.is/uk-biobank

20. Sodini SM, Kemper KE, Wray NR, Trzaskowski M (2018) Comparison of Genotypic and Phenotypic Correlations: Cheverud's Conjecture in Humans. Genetics 209:941-948. https://doi.org/10.1534/genetics.117.300630

21. Campos A, Kho PF, Vazquez-Prada KX et al (2020) Genetic susceptibility to pneumonia: A GWAS meta-analysis between UK Biobank and FinnGen. medRxiv 2020.06.22.20103556. https://doi.org/10.1101/2020.06.22.20103556

22. Noghani T, Rezaeizadeh M, Fazljoo H, Keshavarz SMB (2016) Gastrointestinal Headache; a Narrative Review. Emerg (Tehran) 4:171-183

23. Cámara-Lemarroy CR, Rodriguez-Gutierrez R, Monreal-Robles R, Marfil-Rivera A (2016) Gastrointestinal disorders associated with migraine: A comprehensive review. World J Gastroenterol 22:8149. https://doi.org/10.374 8/wjg.v22.i36.8149

24. Lankarani KB, Akbari M, Tabrizi R (2017) Association of Gastrointestinal Functional Disorders and Migraine Headache: a Population Base Study. Middle East J Dig Dis 9:139-145. https://doi.org/10.15171/mejdd.2017.64

25. Smith J, Parmely JD (2020) Ventral Hernia. In: StatPearls. Treasure Island, StatPearls Publishing

26. Spellar K, Gupta N (2020) Diaphragmatic Hernia. In: StatPearls. Treasure Island, StatPearls Publishing

27. Kavic MS (2005) Hernias as a source of abdominal pain: a matter of concern to general surgeons, gynecologists, and urologists. JSLS 9:249-25

28. Zuber M, Harder F (2001) Benign tumors of the colon and rectum Evidence-Based and Problem-Oriented. Zuckschwerdt, In: Surgical Treatment

29. Mason BN, Russo AF (2018) Vascular Contributions to Migraine: Time to Revisit? Front Cell Neurosci 12:233. https://doi.org/10.3389/fncel.2018.00233

30. Jacobs B, Dussor G (2016) Neurovascular contributions to migraine: Moving beyond vasodilation. Neuroscience 338:130-144. https://doi.org/10.1016/j. neuroscience.2016.06.012

31. Bigal ME, Kurth T, Hu H et al (2009) Migraine and cardiovascular disease: possible mechanisms of interaction. Neurology 72:1864-1871. https://doi. org/10.1212/WNL.0b013e3181a71220

32. Siewert KM, Klarin D, Damrauer SM et al (2020) Cross-trait analyses with migraine reveal widespread pleiotropy and suggest a vascular component to migraine headache. Int J Epidemiol 49:1022-1031. https://doi.org/10.1 093/ije/dyaa050

33. Bansal AB, Khandelwal G (2019) Felodipine. In: StatPearls. Treasure Island StatPearls Publishing

34. Fisch AS, Perry CG, Stephens SH et al (2013) Pharmacogenomics of antiplatelet and anti-coagulation therapy. Curr Cardiol Rep 15:381. https://doi. org/10.1007/s11886-013-0381-3

35. Calcium Channel Blockers (2017) In: LiverTox: Clinical and Research Information on Drug-Induced Liver Injury. National Institute of Diabetes and Digestive and Kidney Diseases, Bethesda (MD). https://www.ncbi.nIm.nih. gov/books/NBK548577/
36. Beavers CJ, Naqvi IA (2020) Clopidogrel. In: StatPearls. Treasure Island, StatPearls Publishing

37. Sarchielli P, Gallai V (2001) Platelets in migraine. J Headache Pain 2:s61-s66. https://doi.org/10.1007/s101940170012

38. Hanington E (1981) Migraine: a platelet disorder. Lancet 318:720-723. https://doi.org/10.1016/S0140-6736(81)91049-7

39. Clougherty JE, Souza K, Cullen MR (2010) Work and its role in shaping the social gradient in health. Ann N Y Acad Sci 1186:102-124. https://doi.org/ 0.1111/j.1749-6632.2009.05338.x

40. Burgard SA, Lin KY (2013) Bad Jobs, Bad Health? How Work and Working Conditions Contribute to Health Disparities. Am Behav Sci 57. https://doi. org/10.1177/0002764213487347

41. Dick FD (2006) Solvent neurotoxicity. Occup Environ Med 63:221-226. https://doi.org/10.1136/oem.2005.022400.

42. Wang JD, Chen JD (1993) Acute and chronic neurological symptoms among paint workers exposed to mixtures of organic solvents. Environ Res 61:107-116. https://doi.org/10.1006/enrs.1993.1054

43. Kim BG, Lee PH, Lee SH et al (2016) Long-Term Effects of Diesel Exhaust Particles on Airway Inflammation and Remodeling in a Mouse Model. Allergy Asthma Immunol Res 8:246-256. https://doi.org/10.4168/aair.2016. 8.3.246

44. Dales RE, Cakmak S, Vidal CB (2009) Air pollution and hospitalization for headache in Chile. Am J Epidemiol 170:1057-1066. https://doi.org/10.1093/a je/kwp217

45. Gerlofs-Nijland ME, van Berlo D, Cassee FR et al (2010) Effect of prolonged exposure to diesel engine exhaust on proinflammatory markers in different regions of the rat brain. Part Fibre Toxicol 7:12. https://doi.org/10.1186/17438977-7-12

46. Marcus DA (2000) Identification of patients with headache at risk of psychological distress. Headache 40:373-376. https://doi.org/10.1046/j.152 6-4610.2000.00056x

47. Peres MFP, Mercante JPP, Tobo PR et al (2017) Anxiety and depression symptoms and migraine: a symptom-based approach research. J Headache Pain 18:37. https://doi.org/10.1186/s10194-017-0742-1

48. Perozzo P, Savi L, Castelli L et al (2005) Anger and emotional distress in patients with migraine and tension-type headache. J Headache Pain 6:392399. https://doi.org/10.1007/s10194-005-0240-8

49. Keyes KM, Pratt C, Galea S et al (2014) The burden of loss: unexpected death of a loved one and psychiatric disorders across the life course in a national study. Am J Psychiatry 171:864-871. https://doi.org/10.1176/appi.a jp.2014.13081132

50. Nicholson RA, Rooney M, Vo K et al (2006) Migraine care among different ethnicities: do disparities exist? Headache 46:754-765. https://doi.org/1 0.1111/j.1526-4610.2006.00453.x

51. Gebhardt M, Kropp P, Hoffmann F, Zettl UK (2019) Headache in the course of multiple sclerosis: a prospective study. J Neural Transm 126:131-139. https://doi.org/10.1007/s00702-018-1959-0

52. Wang J, Zhang B, Shen C et al (2017) Headache symptoms from migraine patients with and without aura through structure-validated self-reports. BMC Neurol 17:193. https://doi.org/10.1186/s12883-017-0973-4

53. Murphy C, Hameed S (2021) Chronic Headaches. In: StatPearls. Treasure Island, StatPearls Publishing

54. Wu Y, Byrne EM, Zheng Z et al (2019) Genome-wide association study of medication-use and associated disease in the UK Biobank. Nat Commun 10: 1891. https://doi.org/10.1038/s41467-019-09572-5

\section{Publisher's Note}

Springer Nature remains neutral with regard to jurisdictional claims in published maps and institutional affiliations. 